\title{
Ultrasound Promoted Green and Facile One-Pot Multicomponent Synthesis of 3,4-dihydropyrano[c]Chromene Derivatives
}

\author{
Ravikumar Nagalapalli, Satyanarayana Reddy Jaggavarapu, Venkata Prasad Jalli, \\ Anand Solomon Kamalakaran, and Gopikrishna Gaddamanugu \\ Department of Chemistry, Sankar Foundation Research Institute, Visakhapatnam 530047. (A.P.), India \\ Correspondence should be addressed to Gopikrishna Gaddamanugu; gkrishnagps@gmail.com
}

Received 27 June 2012; Accepted 29 August 2012

Academic Editor: Davide Vione

Copyright (C) 2013 Ravikumar Nagalapalli et al. This is an open access article distributed under the Creative Commons Attribution License, which permits unrestricted use, distribution, and reproduction in any medium, provided the original work is properly cited.

Ultrasound promoted mild one-pot multicomponent synthesis of 3,4-dihydropyrano[c]chromenes from 4-hydroxycoumarin, arylaldehydes and malononitrile was achieved in aqueous media. The methodology promises advantages of short reaction times, environmentally benign conditions, high yields, and operational convenience.

\section{Introduction}

Dihydropyrano $[c]$ chromene scaffold constitutes an important structural component in several naturally occurring and synthetic molecules displaying broad spectrum of biological activities [1, 2] such as antibacterial [3], antineoplastic activity [4], antimicrobial activity [5], and antiHIV activity [6]. Their biological efficacy is further demonstrated from their applications as cognitive enhancers for the treatment of neurodegenerative Alzheimer's disease [7]. Consequently, numerous elegant synthetic methodologies have been developed for the synthesis of these heterocycles in recent times. Shaker has reported the synthesis of dihydropyrano[ $c]$ chromenes in the presence of organic bases like piperidine/pyridine in ethanol [8], Kidwai documented that microwave irradiation promoted the potassium carbonate catalyzed synthesis [9] of the pyranochromenes while Abdolmohammadi and Saxena et al. have reported diammonium hydrogen phosphate catalyzed synthesis in aqueous ethanol [10]. Shaabani et al. and Shaterian and Oveisi have presented their synthesis in ionic liquids $1,1,3,3-\mathrm{N}, \mathrm{N}, \mathrm{N}^{\prime}, \mathrm{N}^{\prime}$ tetramethylguanidinium trifluoroacetate (TMGT) and 3Hydroxypropanaminium Acetate (HPAA), respectively, [11, 12].

Green chemistry in recent times has gained immense attention and played a significant role in revolutionizing synthetic organic chemistry to minimize the usage of organic solvents and their disposal thereby developing environmentfriendly organic synthesis [13]. In this context, water has been explored in several synthetic methodologies as an efficient solvent which successfully displaced the usage of harmful organic solvents thereby reducing the generation of hazardous wastes [14]. On the other hand, ultrasound promoted reactions paved way for the modification and improvement of several existing synthetic methodologies by enhancing their reaction rates $[15,16]$ thus appending considerable value addition to the green chemistry procedures. The cavitation energy produced during the process of ultrasonication paves way for alternative pathways due to the formation of high energy intermediates thereby modifying and improving the reaction rates [17].

\section{Results and Discussion}

Earlier, we have reported ultrasound promoted asymmetric Mannich reaction and chromeno-quinolines synthesis $[18,19]$. In continuation of our efforts to develop green chemistry protocols and foreseeing the importance of 3,4dihydropyrano $[c]$ chromenes synthesis, we have developed a mild and efficient procedure for their synthesis under 


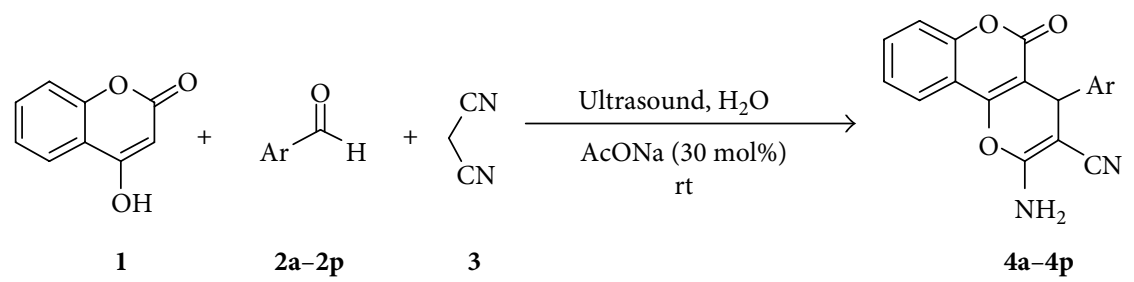

SCHeme 1: Synthetic route for the synthesis of 3,4-dihydropyrano[c]chromene derivatives $\mathbf{4 a - 4 p}$. Reagents and conditions: Sodium acetate (30 $\mathrm{mol} \%), \mathrm{H}_{2} \mathrm{O}$, Ultrasound irradiation, and room temperature.

TABLE 1: Optimization of reaction conditions with different solvents and catalysts for the synthesis of $4 a^{a}$.

\begin{tabular}{|c|c|c|c|c|c|}
\hline Entry & Catalyst & Solvent & $\operatorname{Mol}(\%)$ & Time (min) & Yield $(\%)^{\mathrm{b}}$ \\
\hline 1 & Trisodium citrate & $\mathrm{H}_{2} \mathrm{O}$ & 30 & 10 & 57 \\
\hline 2 & $\mathrm{NH}_{4} \mathrm{OAc}$ & $\mathrm{H}_{2} \mathrm{O}$ & 30 & 10 & 70 \\
\hline 3 & L-proline & $\mathrm{H}_{2} \mathrm{O}$ & 30 & 10 & 30 \\
\hline 4 & Nafion-H & $\mathrm{H}_{2} \mathrm{O}$ & $50 \mathrm{mg}$ & 10 & 40 \\
\hline 5 & Amberlyst & $\mathrm{H}_{2} \mathrm{O}$ & $50 \mathrm{mg}$ & 10 & 30 \\
\hline 6 & Sodium acetate & $\mathrm{H}_{2} \mathrm{O}$ & 30 & 10 & 92 \\
\hline 7 & Sodium acetate & PEG & 30 & 10 & 50 \\
\hline 8 & Sodium acetate & DMSO & 30 & 10 & 36 \\
\hline 9 & Sodium acetate & $\mathrm{DMF}$ & 30 & 10 & 28 \\
\hline 10 & Sodium acetate & $\mathrm{EtOH}$ & 30 & 10 & 70 \\
\hline 11 & Sodium acetate & $\mathrm{H}_{2} \mathrm{O}$ & 30 & 180 & $30^{\mathrm{c}}$ \\
\hline 12 & - & $\mathrm{H}_{2} \mathrm{O}$ & - & 30 & $35^{\mathrm{d}}$ \\
\hline
\end{tabular}

${ }^{a}$ The reactions were carried out at room temperature using 4-hydroxycoumarin $(1 \mathrm{mmol})$, benzaldehyde $(1 \mathrm{mmol})$, malononitrile $(1.2 \mathrm{mmol})$, and catalyst $(30 \mathrm{~mol} \%)$ in water $(5 \mathrm{~mL})$ under ultrasound conditions, ${ }^{\mathrm{b}}$ Yields refer to the precipitated products, ${ }^{\mathrm{c}}$ absence of ultrasound conditions, ${ }^{\mathrm{d}}$ Only ultrasound, no catalyst.

ultrasound conditions using sodium acetate as a catalyst in aqueous conditions at room temperature (Scheme 1).

In order to optimize the reaction conditions, the synthesis of 2-amino-5-oxo-4-phenyl-4,5-dihydropyrano[3,2c]chromene-3-carbonitrile (4a) from the condensation of 4-hydroxycoumarin, benzaldehyde, and malononitrile was studied in the presence of a variety of catalysts and solvents under ultrasound conditions. As shown in Table 1, ultrasonication promotes the formation of products with varying yields and reaction rates under different catalytic systems. First, a trial reaction was performed in water with $30 \mathrm{~mol} \%$ of trisodium citrate $\left(\mathrm{Na}_{3} \mathrm{C}_{6} \mathrm{H}_{5} \mathrm{O}_{7}\right)$ as a promoter (Table 1 , entry 1). It was observed that after 10 minutes of the ultrasound irradiation the required product was obtained in 57\% yield. Among various other catalysts screened ammonium acetate, L-proline, Nafion-H, and Amberlyst afforded poorto-moderate yields (30-70\%), while sodium acetate ( $\mathrm{NaOAc})$ gave $92 \%$ yield of the desired product (Table 1,entry 2-6) in just 10 minutes. To validate the choice of solvent, various solvents were screened to check their influence on the rates and yields of the reaction (Table 1, entries 7-10). Solvents such as PEG, DMSO, and DMF afforded poor-to-moderate yields $(28-50 \%)$, while better yields of the products were observed when the reaction was conducted in ethanol (70\%). Further to authenticate the effect of ultrasound conditions, a reaction was conducted in absence of ultrasound which resulted in only $30 \%$ of desired product after stirring for
3 hours (Table 1, Entry 11). Surprisingly, ultrasonication alone in absence of catalyst was able to promote the reaction affording the product in $35 \%$ yield after of 30 minutes (Table 1, Entry 12). The best yield was obtained when the reaction was performed with sodium acetate as a catalyst in water under ultrasonication conditions.

With the above optimized reaction conditions, a series of 3,4-dihyropyrano[ $c$ ] chromene derivatives $4 \mathbf{a}-\mathbf{4} \mathbf{p}$ were synthesized (Table 2). As shown in Table 2, when halogen substituted aromatic aldehydes such as 2-chloro, 3-chloro, 2,4-dichloro, 2,3-dichloro, 4-chloro, 4-bromo, and 4-fluoro substrates were employed under the reaction conditions excellent yields (82-94\%) of the corresponding products $4 \mathbf{b}-$ 4 h were obtained (Table 2, entries 2-8). Aromatic aldehydes possessing electron donating substituents such as 2-methyl, 4-methyl, 4-methoxy, 3,4-dimethoxy, and 2,5-dimethoxy have afforded the pyranochromenes $4 \mathbf{i}-\mathbf{4 m}$ in $76-92 \%$ yields (Table 2, entries 9-13). Similarly, electron withdrawing aromatic aldehydes possessing 3-nitro and 4-nitro groups also afforded the pyranochromene derivatives $\mathbf{4 n - 4 0}$ in $85 \%$ and $94 \%$ yields, respectively, (Table 2, entries 14 and 15). Finally, p-hydroxybenzaldehyde possessing phenol group also afforded the required product $\mathbf{4 p}$ in $91 \%$ yield (Table 2 , entry 16). All the above reactions were time optimized and products were obtained as precipitates from the reaction mixtures in pure form which evaded the tedious column purification procedures. From the above observations it is 
TABLE 2: Ultrasound promoted synthesis of 3,4-Dihyropyrano[c]chromenes $\mathbf{4 a - 4} \mathbf{p}$ using NaOAc as a catalyst ${ }^{\mathrm{a}}$.

\begin{tabular}{|c|c|c|c|c|c|}
\hline Entry & $\mathrm{Ar}^{\mathrm{b}}$ & Product & Time (min) & Yield $(\%)^{c}$ & Melting point $\left({ }^{\circ} \mathrm{C}\right) /$ (lit.) \\
\hline 1 & & $4 a$ & 10 & 92 & $256-259 /(258-260)[8]$ \\
\hline 2 & & $4 \mathrm{~b}$ & 8 & 85 & $264-266 /(266-268)[20]$ \\
\hline 3 & & $4 \mathrm{c}$ & 6 & 82 & $246-248 /(245-247)[21]$ \\
\hline 4 & & 4d & 5 & 86 & $255-257 /(257-259)[10]$ \\
\hline 5 & & $4 \mathrm{e}$ & 6 & 76 & $282-284 /(280-282)[10]$ \\
\hline 6 & & $4 \mathrm{f}$ & 5 & 94 & $256-258 /(258-260)[8]$ \\
\hline 7 & & $4 \mathrm{~g}$ & 7 & 82 & $247-249 /(249-251)[22]$ \\
\hline
\end{tabular}


TABLE 2: Continued.

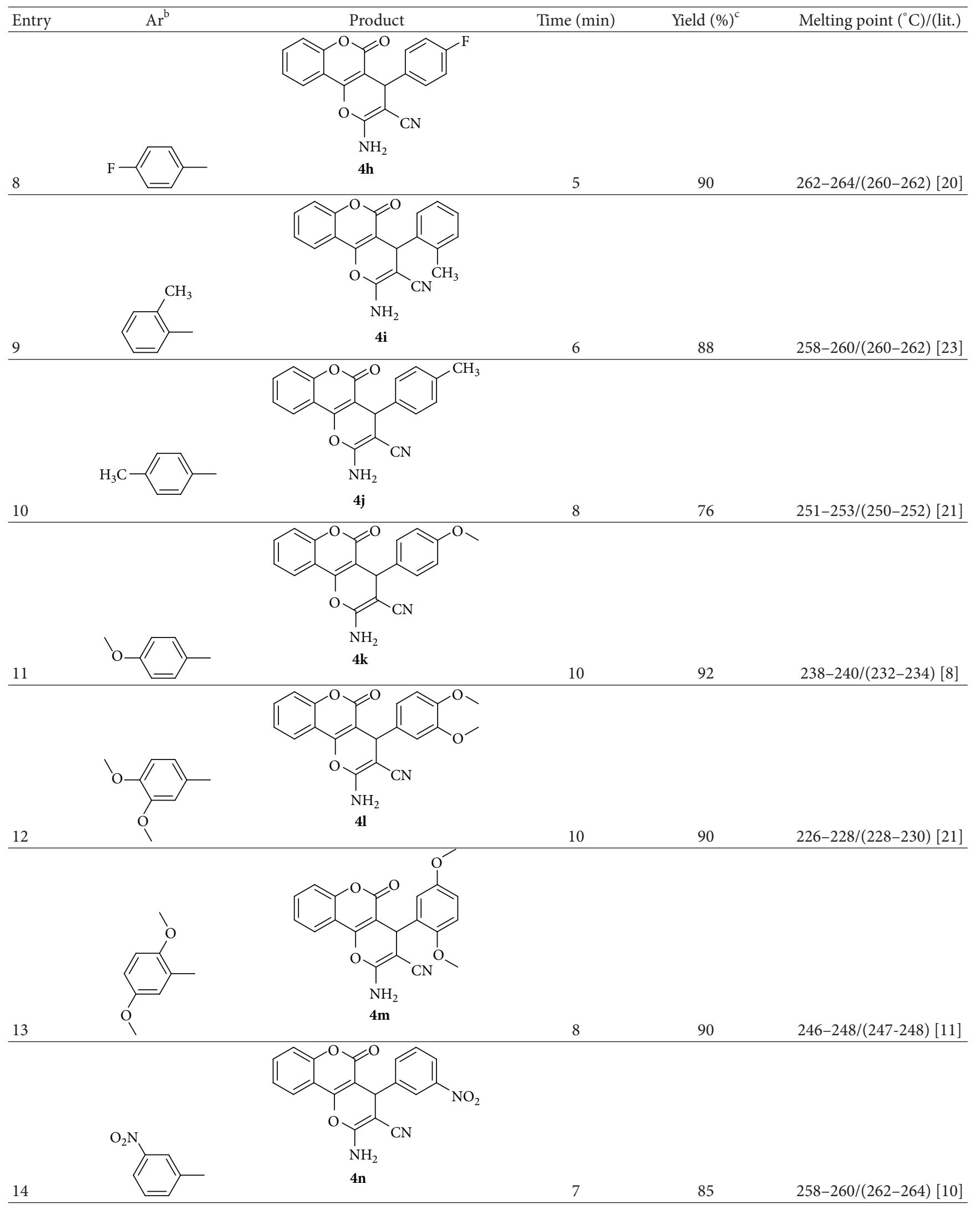


TABLE 2: Continued.

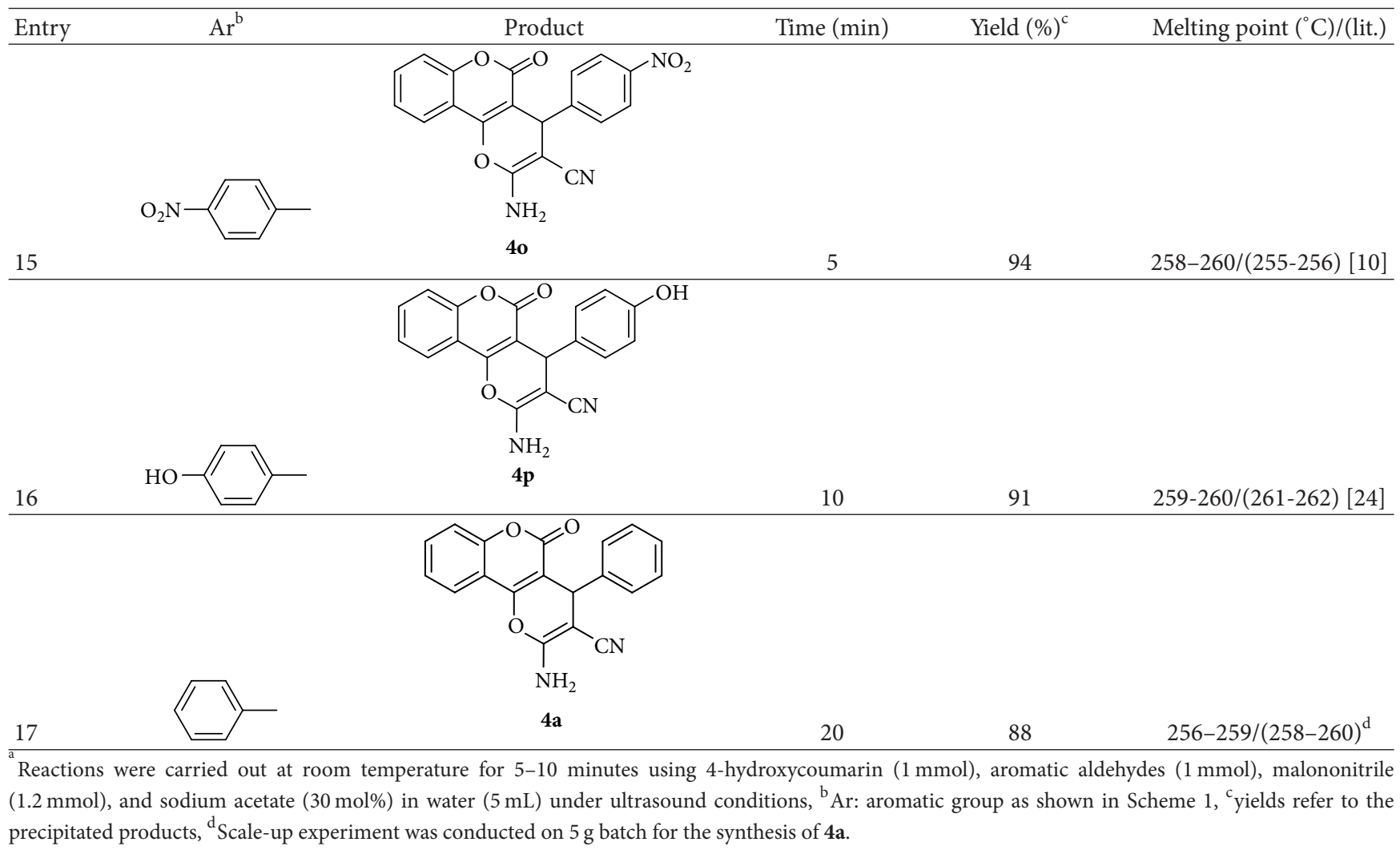

evident that the ultrasound promoted protocol has successfully accommodated wide range of aromatic aldehydes possessing broad array of functional groups and substitution patterns.

To further explore the efficacy of ultrasound methodology on the present methodology a scale-up experiment was conducted on $5 \mathrm{~g}$ batch for the synthesis of $4 \mathbf{a}$. Gratifyingly, we observed that the ultrasound methodology provides $88 \%$ of $\mathbf{4 a}$ (Table 2, entry 17) as a pure precipitate with a slight compromise in time (20 minutes).

\section{Conclusion}

In conclusion, we have developed a simple, efficient, and green protocol for the synthesis of 3,4-dihydropyrano $[c]$ chromenes in high yields promoted by ultrasound conditions in aqueous medium. The results also suggest that the present methodology can be effectively applied for the synthesis of the products in grams scale. Finally, the operationally simple protocol did not require any column purifications as the products were isolated as pure precipitates from the reaction mixture.

\section{Experimental}

All reagents were purchased from Merck and Avra synthesis Pvt. Ltd. and used without further purification. All yields refer to the precipitated products. All the products were characterized by ${ }^{1} \mathrm{H}$ NMR, ${ }^{13} \mathrm{C}$ NMR, IR, and EI-Mass analysis and were found to be in good agreement with those reported in the literature. The ${ }^{1} \mathrm{H}$ NMR $(500 \mathrm{MHz})$ and ${ }^{13} \mathrm{C}$ NMR $(125 \mathrm{MHz})$ were recorded on a Bruker Avance DPX $500 \mathrm{MHz}$ instrument at room temperature in DMSO using TMS as an internal reference. IR spectra were recorded on a Bruker $\alpha$ alpha-T spectrophotometer. Melting points were determined by NETZSCH DSC 200 instrument. Sonication was performed in Leelasonic ultrasonic reactor with a frequency of $40 \mathrm{KHz}$ and a nominal power of $250 \mathrm{~W}$.

4.1. General Procedure for the Synthesis of Dihydropyrano[c]chromene Derivatives 4a-p. A solution of 4-hydroxy$\begin{array}{llll}\text { coumarin } & \mathbf{1} & (1 \mathrm{mmol}), & \text { aromatic }\end{array}$ aldehydes $\quad \mathbf{2 a}-\mathbf{p} \quad(1 \mathrm{mmol}), \quad$ malononitrile 3 ( $1.2 \mathrm{mmol})$, and sodium acetate $(30 \mathrm{~mol} \%)$ in $\mathrm{H}_{2} \mathrm{O}$ was sonicated at room temperature for required time (Table 2). After completion of the reaction as monitored by TLC the precipitate obtained was filtered and washed with aqueous ethanol to give pure products. All the obtained products were characterized by comparing their physical data with the authentic samples.

2-Amino-5-oxo-4-phenyl-4,5-dihydropyrano[3,2-c] chromene-3-carbonitrile 4a. M.p. 256-259 $\mathrm{C}$ [lit: 258-260 ${ }^{\circ} \mathrm{C}$ ] [8]. ${ }^{1} \mathrm{H}$ NMR $\left(500 \mathrm{MHz}, \mathrm{DMSO}_{-} \mathrm{d}_{6}\right): \delta 4.36(1 \mathrm{H}, \mathrm{s}, \mathrm{CH})$, $7.23\left(2 \mathrm{H}, \mathrm{d}, J=7.8 \mathrm{~Hz}, \mathrm{H}_{\mathrm{Ar}}\right), 7.29\left(1 \mathrm{H}\right.$, br s, $\left.\mathrm{H}_{\mathrm{Ar}}\right), 7.33$ 
$\left(2 \mathrm{H}, \mathrm{t}, J=7.5 \mathrm{~Hz}, \mathrm{H}_{\mathrm{Ar}}\right), 7.32\left(2 \mathrm{H}\right.$, br s, $\left.\mathrm{NH}_{2}\right), 7.45(1 \mathrm{H}$, $\left.\mathrm{d}, J=8.4 \mathrm{~Hz}, \mathrm{H}_{\mathrm{Ar}}\right), 7.49\left(1 \mathrm{H}, \mathrm{t}, J=7.6 \mathrm{~Hz}, \mathrm{H}_{\mathrm{Ar}}\right), 7.71(1 \mathrm{H}$, $\left.\mathrm{t}, \mathrm{J}=7.5 \mathrm{~Hz}, \mathrm{H}_{\mathrm{Ar}}\right), 7.91\left(1 \mathrm{H}, \mathrm{d}, J=7.8 \mathrm{~Hz}, \mathrm{H}_{\mathrm{Ar}}\right) \mathrm{ppm} .{ }^{13} \mathrm{C}$ NMR (125 MHz, DMSO-d $\left.{ }_{6}\right): \delta 58.18,104.88,114.84,117.44$, $121.10,123.34,125.64,127.99,128.50,129.59,132.79,145.21$, $153.56,154.79,158.86,161.41 \mathrm{ppm}$. IR $\left(\mathrm{KBr}, \mathrm{cm}^{-1}\right): 3378$, $3286,3178,2196,1709,1674,1604$. EI-MS: $\mathrm{m} / \mathrm{z}=316.06$.

2-Amino-4-(2-chlorophenyl)-5-oxo-4,5-dihydropyrano[3,2-c] chromene-3-carbonitrile4b. M.p. $264-266^{\circ} \mathrm{C}$ [lit: $266-268^{\circ} \mathrm{C}$ ] [20]. ${ }^{1} \mathrm{H}$ NMR $\left(500 \mathrm{MHz}, \mathrm{DMSO}-\mathrm{d}_{6}\right): \delta 4.48(1 \mathrm{H}, \mathrm{s}, \mathrm{CH})$, $7.15\left(1 \mathrm{H}, \mathrm{d}, J=7.8 \mathrm{~Hz}, \mathrm{H}_{\mathrm{Ar}}\right), 7.54\left(1 \mathrm{H}, \mathrm{d}, J=7.8 \mathrm{~Hz}, \mathrm{H}_{\mathrm{Ar}}\right)$, $7.22\left(2 \mathrm{H}, \mathrm{t}, J=7.5 \mathrm{~Hz}, \mathrm{H}_{\mathrm{Ar}}\right), 7.25\left(2 \mathrm{H}\right.$, br s, $\left.\mathrm{NH}_{2}\right), 7.42$ $\left(1 \mathrm{H}, \mathrm{d}, J=8.4 \mathrm{~Hz}, \mathrm{H}_{\mathrm{Ar}}\right), 7.55\left(1 \mathrm{H}, \mathrm{t}, J=7.6 \mathrm{~Hz}, \mathrm{H}_{\mathrm{Ar}}\right), 7.61$ $\left(1 \mathrm{H}, \mathrm{t}, J=7.5 \mathrm{~Hz}, \mathrm{H}_{\mathrm{Ar}}\right), 7.89\left(1 \mathrm{H}, \mathrm{d}, J=7.8 \mathrm{~Hz}, \mathrm{H}_{\mathrm{Ar}}\right) \mathrm{ppm}$. ${ }^{13} \mathrm{C}$ NMR (125 MHz, DMSO- $\left.\mathrm{d}_{6}\right):{ }^{13} \mathrm{C} \mathrm{NMR}(125 \mathrm{MHz}$, DMSO- $\left.\mathrm{d}_{6}\right): \delta 57.65,105.40,112.80,118.34,119.64,122.38$, $126.42,128.38,131.75,132.85,135.75,143.12,152.06,155.62$, $157.73,161.54 \mathrm{ppm}$. IR $\left(\mathrm{KBr}, \mathrm{cm}^{-1}\right): 3374,3283,3175,2192$, $1710,1672,1605$. EI-MS: $\mathrm{m} / \mathrm{z}=350.04$.

2-Amino-4-(3-chlorophenyl)-5-oxo-4,5-dihydropyrano[3,2-c] chromene-3-carbonitrile 4c. M.p. $246-248^{\circ} \mathrm{C}$ [lit: $245-247^{\circ} \mathrm{C}$ ] [21]. ${ }^{1} \mathrm{H}$ NMR $\left(500 \mathrm{MHz}, \mathrm{DMSO}-\mathrm{d}_{6}\right): \delta 4.54(1 \mathrm{H}, \mathrm{s}, \mathrm{CH})$, $7.34\left(1 \mathrm{H}, \mathrm{d}, J=6.7 \mathrm{~Hz}, \mathrm{H}_{\mathrm{Ar}}\right), 7.41\left(1 \mathrm{H}, \mathrm{t}, J=7.6 \mathrm{~Hz}, \mathrm{H}_{\mathrm{Ar}}\right)$, $7.66\left(2 \mathrm{H}\right.$, br s, $\left.\mathrm{NH}_{2}\right), 7.74\left(1 \mathrm{H}, \mathrm{t}, J=7.6 \mathrm{~Hz}, \mathrm{H}_{\mathrm{Ar}}\right), 7.54$ $\left(1 \mathrm{H}, \mathrm{dt}, J=7.5,1.3 \mathrm{~Hz}, \mathrm{H}_{\mathrm{Ar}}\right), 7.68\left(1 \mathrm{H}, \mathrm{d}, J=6.8 \mathrm{~Hz}, \mathrm{H}_{\mathrm{Ar}}\right)$, $7.89\left(1 \mathrm{H}, \mathrm{dd}, J=6.8,1.2 \mathrm{~Hz}, \mathrm{H}_{\mathrm{Ar}}\right), 8.12(1 \mathrm{H}, \mathrm{dd}, J=8.4$, $\left.1.4 \mathrm{~Hz}, \mathrm{H}_{\mathrm{Ar}}\right), 8.14\left(1 \mathrm{H}, \mathrm{s}, \mathrm{H}_{\mathrm{Ar}}\right) \mathrm{ppm} .{ }^{13} \mathrm{C} \mathrm{NMR}(125 \mathrm{MHz}$, DMSO $\left.-\mathrm{d}_{6}\right): \delta 58.62,104.74,113.81,117.32,119.73,121.13$, $123.24,123.64,125.67,131.92,134.24,135.63,145.36,147.62$, $152.43,154.65,159.03,160.46 \mathrm{ppm}$. IR $\left(\mathrm{KBr}, \mathrm{cm}^{-1}\right): 3404$, $3322,3183,2198,1706,1674,1531,1349$. EI-MS: $\mathrm{m} / \mathrm{z}=$ 350.05 .

2-Amino-4-(2,4-dichlorophenyl)-5-oxo-4,5-dihydropyrano[3, 2-c]chromene-3-carbonitrile 4d. M.p. 255-257 $\mathrm{C}$ [lit: 257$259^{\circ} \mathrm{C}$ ] [10]. ${ }^{1} \mathrm{H}$ NMR (500 MHz, DMSO-d 6 ): $\delta 4.99(1 \mathrm{H}$, s, CH), $7.36\left(1 \mathrm{H}, \mathrm{dd}, J=8.3,1.9 \mathrm{~Hz}, \mathrm{H}_{\mathrm{Ar}}\right), 7.40(1 \mathrm{H}$, $\left.\mathrm{d}, J=8.3 \mathrm{~Hz}, \mathrm{H}_{\mathrm{Ar}}\right), 7.41\left(2 \mathrm{H}\right.$, br s, $\left.\mathrm{NH}_{2}\right), 7.46(1 \mathrm{H}$, $\left.\mathrm{d}, J=8.3 \mathrm{~Hz}, \mathrm{H}_{\mathrm{Ar}}\right), 7.51\left(1 \mathrm{H}, \mathrm{t}, J=7.7 \mathrm{~Hz}, \mathrm{H}_{\mathrm{Ar}}\right), 7.56(1 \mathrm{H}$, $\left.\mathrm{d}, J=2.1 \mathrm{~Hz}, \mathrm{H}_{\mathrm{Ar}}\right), 7.73\left(1 \mathrm{H}, \mathrm{t}, J=8.2 \mathrm{~Hz}, \mathrm{H}_{\mathrm{Ar}}\right), 7.92(1 \mathrm{H}$, d, $\left.J=8.9 \mathrm{~Hz}, \mathrm{H}_{\mathrm{Ar}}\right)$ ppm. ${ }^{13} \mathrm{C} \mathrm{NMR}\left(125 \mathrm{MHz}, \mathrm{DMSO}-\mathrm{d}_{6}\right): \delta$ 57.10, 103.38, 113.71, 117.47, 119.43, 123.42, 125.57, 128.71, $129.73,132.95,133.28,133.96,134.28,140.26,153.14,155.05$, $159.05,160.23$ ppm. IR (KBr, cm $\left.{ }^{-1}\right): 3463,3295,3163,3070$, 2198, 1715, 1674, 1590. EI-MS: $\mathrm{m} / \mathrm{z}=384.02$.

2-Amino-4-(2,3-dichlorophenyl)-5-oxo-4,5-dihydropyrano[3, 2-c]chromene-3-carbonitrile 4e. M.p. $282-284^{\circ} \mathrm{C}$ [lit: 280$\left.282^{\circ} \mathrm{C}\right] \quad[10] .{ }^{1} \mathrm{H}$ NMR $\left(500 \mathrm{MHz}, \mathrm{DMSO}_{-} \mathrm{d}_{6}\right): \delta 4.86$ $(1 \mathrm{H}, \mathrm{s}, \mathrm{CH}), 7.36\left(1 \mathrm{H}, \mathrm{dd}, J=8.3,1.9 \mathrm{~Hz}, \mathrm{H}_{\mathrm{Ar}}\right), 7.40$ $\left(1 \mathrm{H}, \mathrm{d}, J=8.3 \mathrm{~Hz}, \mathrm{H}_{\mathrm{Ar}}\right), 7.41\left(2 \mathrm{H}\right.$, br s, $\left.\mathrm{NH}_{2}\right), 7.46(1 \mathrm{H}$, $\left.\mathrm{d}, J=8.3 \mathrm{~Hz}, \mathrm{H}_{\mathrm{Ar}}\right), 7.51\left(1 \mathrm{H}, \mathrm{t}, J=7.7 \mathrm{~Hz}, \mathrm{H}_{\mathrm{Ar}}\right), 7.56(1 \mathrm{H}$, $\left.\mathrm{d}, J=2.1 \mathrm{~Hz}, \mathrm{H}_{\mathrm{Ar}}\right), 7.73\left(1 \mathrm{H}, \mathrm{t}, J=8.2 \mathrm{~Hz}, \mathrm{H}_{\mathrm{Ar}}\right), 7.92(1 \mathrm{H}$, $\left.\mathrm{d}, J=8.9 \mathrm{~Hz}, \mathrm{H}_{\mathrm{Ar}}\right) \mathrm{ppm}^{13} \mathrm{C}$ NMR $\left(125 \mathrm{MHz}, \mathrm{DMSO}-\mathrm{d}_{6}\right): \delta$ $57.10,103.38,113.71,117.47,119.43,123.42,125.57,128.71$,
$129.73,132.95,133.28,133.96,134.28,140.26,153.14,155.05$, $159.05,160.23 \mathrm{ppm}$. IR $\left(\mathrm{KBr}, \mathrm{cm}^{-1}\right): 3463,3295,3163,3070$, 2198, 1715, 1674, 1590. EI-MS: $\mathrm{m} / \mathrm{z}=384.02$.

2-Amino-4-(4-chlorophenyl)-5-oxo-4,5-dihydropyrano[3,2-c] chromene-3-carbonitrile 4f. M.p. $256-258^{\circ} \mathrm{C}$ [lit: $258-260^{\circ} \mathrm{C}$ ] [8]. ${ }^{1} \mathrm{H}$ NMR (500 MHz, DMSO-d 6 ): $\delta 4.50(1 \mathrm{H}, \mathrm{s}, \mathrm{CH}), 7.32$ $\left(2 \mathrm{H}, \mathrm{d}, J=8.2 \mathrm{~Hz}, \mathrm{H}_{\mathrm{Ar}}\right), 7.26\left(2 \mathrm{H}\right.$, br s, $\left.\mathrm{NH}_{2}\right), 7.34(2 \mathrm{H}$, br s, $\left.\mathrm{H}_{\mathrm{Ar}}\right), 7.34\left(1 \mathrm{H}, \mathrm{d}, J=8.2 \mathrm{~Hz}, \mathrm{H}_{\mathrm{Ar}}\right), 7.58(1 \mathrm{H}, \mathrm{t}, J=7.6 \mathrm{~Hz}$, $\left.\mathrm{H}_{\mathrm{Ar}}\right), 7.78\left(1 \mathrm{H}, \mathrm{t}, J=7.8 \mathrm{~Hz}, \mathrm{H}_{\mathrm{Ar}}\right), 7.92(1 \mathrm{H}, \mathrm{d}, J=7.8 \mathrm{~Hz}$, $\mathrm{H}_{\mathrm{Ar}}$ ) ppm. ${ }^{13} \mathrm{C}$ NMR (125 MHz, DMSO-d 6 ): $\delta$ 58.65, 104.40, $113.80,117.34,119.86,123.38,125.42,129.28,130.45,132.65$, $133.75,143.12,153.06,154.42,158.93,160.34$ ppm. IR $(\mathrm{KBr}$, $\left.\mathrm{cm}^{-1}\right): 3380,3318,3179,2194,1715,1665,1605$. EI-MS: m/z $=350.05$.

2-Amino-4-(4-bromophenyl)-5-oxo-4,5-dihydropyrano[3,2-c] chromene-3-carbonitrile 4g. M.p. $247-249^{\circ} \mathrm{C}$ [lit: 249-251 ${ }^{\circ} \mathrm{C}$ ] [22]. ${ }^{1} \mathrm{H}$ NMR (500 MHz, DMSO-d 6 ): $\delta 4.42(1 \mathrm{H}, \mathrm{s}, \mathrm{CH})$, $7.35\left(2 \mathrm{H}, \mathrm{d}, J=8.2 \mathrm{~Hz}, \mathrm{H}_{\mathrm{Ar}}\right), 7.28\left(2 \mathrm{H}\right.$, br s, $\left.\mathrm{NH}_{2}\right), 7.42$ $\left(2 \mathrm{H}\right.$, br s, $\left.\mathrm{H}_{\mathrm{Ar}}\right), 7.38\left(1 \mathrm{H}, \mathrm{d}, J=8.2 \mathrm{~Hz}, \mathrm{H}_{\mathrm{Ar}}\right), 7.62(1 \mathrm{H}$, $\left.\mathrm{t}, J=7.6 \mathrm{~Hz}, \mathrm{H}_{\mathrm{Ar}}\right), 7.88\left(1 \mathrm{H}, \mathrm{t}, J=7.8 \mathrm{~Hz}, \mathrm{H}_{\mathrm{Ar}}\right), 7.98(1 \mathrm{H}$, $\left.\mathrm{d}, J=7.8 \mathrm{~Hz}, \mathrm{H}_{\mathrm{Ar}}\right)$ ppm. ${ }^{13} \mathrm{C}$ NMR $\left(125 \mathrm{MHz}, \mathrm{DMSO}-\mathrm{d}_{6}\right): \delta$ $57.45,102.50,113.78,116.54,118.76,122.68,124.42,129.68$, $131.54,133.45,134.85,144.42,154.76,155.62,159.32$, 160.34 ppm. IR $\left(\mathrm{KBr}, \mathrm{cm}^{-1}\right): 3380,3318,3179,2194,1715$, 1665, 1605. EI-MS: $\mathrm{m} / \mathrm{z}=394.01$.

2-Amino-4-(4-fluorophenyl)-5-oxo-4,5-dihydropyrano[3,2-c] chromene-3-carbonitrile 4h. M.p. 262-264 ${ }^{\circ} \mathrm{C}$ [lit: 260-262 ${ }^{\circ} \mathrm{C}$ ] [20]. ${ }^{1} \mathrm{H}$ NMR $\left(500 \mathrm{MHz}, \mathrm{DMSO}-\mathrm{d}_{6}\right): \delta 4.47(1 \mathrm{H}, \mathrm{s}, \mathrm{CH})$, $7.45\left(2 \mathrm{H}, \mathrm{d}, J=8.2 \mathrm{~Hz}, \mathrm{H}_{\mathrm{Ar}}\right), 7.38\left(2 \mathrm{H}\right.$, br s, $\left.\mathrm{NH}_{2}\right), 7.54$ $\left(2 \mathrm{H}\right.$, br s, $\left.\mathrm{H}_{\mathrm{Ar}}\right), 7.46\left(1 \mathrm{H}, \mathrm{d}, J=8.2 \mathrm{~Hz}, \mathrm{H}_{\mathrm{Ar}}\right), 7.72(1 \mathrm{H}$, $\left.\mathrm{t}, J=7.6 \mathrm{~Hz}, \mathrm{H}_{\mathrm{Ar}}\right), 7.82\left(1 \mathrm{H}, \mathrm{t}, J=7.8 \mathrm{~Hz}, \mathrm{H}_{\mathrm{Ar}}\right), 7.96(1 \mathrm{H}$, $\left.\mathrm{d}, J=7.8 \mathrm{~Hz}, \mathrm{H}_{\mathrm{Ar}}\right)$ ppm. ${ }^{13} \mathrm{C} \mathrm{NMR}\left(125 \mathrm{MHz}, \mathrm{DMSO}-\mathrm{d}_{6}\right): \delta$ $56.24,105.40,114.80,118.34,119.86,124.38,125.42,129.76$, $131.25,132.98,135.75,144.12,153.56,154.82,157.93$, $161.34 \mathrm{ppm}$. IR (KBr, cm $\left.{ }^{-1}\right): 3378,3294,2192,1714,1679$, 1605, 1507, 1378. EI-MS: $\mathrm{m} / \mathrm{z}=334.06$.

2-Amino-5-oxo-4-o-tolyl-4,5-dihydropyrano[3,2-c]chromene3-carbonitrile 4i. M.p. $258-260^{\circ} \mathrm{C}$ [lit: $260-262^{\circ} \mathrm{C}$ ] [23]. ${ }^{1} \mathrm{H}$ NMR $\left(500 \mathrm{MHz}, \mathrm{DMSO}-\mathrm{d}_{6}\right): \delta 2.36\left(3 \mathrm{H}, \mathrm{s}, \mathrm{CH}_{3}\right), 4.28(1 \mathrm{H}$, s, CH), $7.16\left(1 \mathrm{H}, \mathrm{d}, J=7.8 \mathrm{~Hz}, \mathrm{H}_{\mathrm{Ar}}\right), 7.64(1 \mathrm{H}, \mathrm{d}, J=7.8 \mathrm{~Hz}$, $\left.\mathrm{H}_{\mathrm{Ar}}\right), 7.32\left(2 \mathrm{H}, \mathrm{t}, J=7.5 \mathrm{~Hz}, \mathrm{H}_{\mathrm{Ar}}\right), 7.38\left(2 \mathrm{H}\right.$, br s, $\left.\mathrm{NH}_{2}\right)$, $7.46\left(1 \mathrm{H}, \mathrm{d}, J=8.4 \mathrm{~Hz}, \mathrm{H}_{\mathrm{Ar}}\right), 7.58\left(1 \mathrm{H}, \mathrm{t}, J=7.6 \mathrm{~Hz}, \mathrm{H}_{\mathrm{Ar}}\right)$, $7.68\left(1 \mathrm{H}, \mathrm{t}, J=7.5 \mathrm{~Hz}, \mathrm{H}_{\mathrm{Ar}}\right), 7.78(1 \mathrm{H}, \mathrm{d}, J=7.8 \mathrm{~Hz}$, $\left.\mathrm{H}_{\mathrm{Ar}}\right) \mathrm{ppm}^{13} \mathrm{C} \mathrm{NMR}\left(125 \mathrm{MHz}, \mathrm{DMSO}-\mathrm{d}_{6}\right): \delta 56.95,107.40$, $116.80,118.34,119.34,124.38,127.42,128.38,131.65,134.85$, $135.75,146.12,153.06,155.62,157.73,162.54$ ppm. IR (KBr, $\left.\mathrm{cm}^{-1}\right): 3378,3284,3176,2194,1710,1672,1605$.EI-MS: m/z $=330.09$.

2-Amino-5-oxo-4-p-tolyl-4,5-dihydropyrano[3,2-c]chromene3-carbonitrile 4j. (Table 2 entry 10). M.p. $251-253^{\circ} \mathrm{C}$ [lit: 250-252 ${ }^{\circ} \mathrm{C}$ ] [21]. ${ }^{1} \mathrm{H}$ NMR $\left(500 \mathrm{MHz}, \mathrm{DMSO}-\mathrm{d}_{6}\right): \delta 2.38$ $\left(3 \mathrm{H}, \mathrm{s}, \mathrm{CH}_{3}\right), 4.38(1 \mathrm{H}, \mathrm{s}, \mathrm{CH}), 7.34\left(2 \mathrm{H}, \mathrm{d}, J=8.2 \mathrm{~Hz}, \mathrm{H}_{\mathrm{Ar}}\right)$, $7.45\left(2 \mathrm{H}\right.$, br s, $\left.\mathrm{NH}_{2}\right), 7.38\left(2 \mathrm{H}\right.$, br s, $\left.\mathrm{H}_{\mathrm{Ar}}\right), 7.34(1 \mathrm{H}, \mathrm{d}, J=8.2$, $\left.\mathrm{Hz}, \mathrm{H}_{\mathrm{Ar}}\right), 7.58\left(1 \mathrm{H}, \mathrm{t}, J=7.6 \mathrm{~Hz}, \mathrm{H}_{\mathrm{Ar}}\right), 7.78(1 \mathrm{H}, \mathrm{t}, J=7.8 \mathrm{~Hz}$, 
$\left.\mathrm{H}_{\mathrm{Ar}}\right), 7.82\left(1 \mathrm{H}, \mathrm{d}, J=7.8 \mathrm{~Hz}, \mathrm{H}_{\mathrm{Ar}}\right) \mathrm{ppm} .{ }^{13} \mathrm{C} \mathrm{NMR}(125 \mathrm{MHz}$, DMSO- $\left.\mathrm{d}_{6}\right): \delta 59.65,103.40,115.80,118.34,119.86,122.38$, $125.42,129.28,130.45,131.65,133.75,144.12,153.06,154.42$, 158.93, 160.34 ppm. IR (KBr, cm $\left.{ }^{-1}\right): 3378,3296,2192,1710$, 1676, 1604, 1510, 1378. EI-MS: $\mathrm{m} / \mathrm{z}=330.10$.

2-Amino-4-(4-methoxyphenyl)-5-oxo-4,5-dihydropyrano[3, 2-c]chromene-3-carbonitrile 4k. M.p. 238-240 $\mathrm{C}$ [lit: 232$234^{\circ} \mathrm{C}$ ] [8]. ${ }^{1} \mathrm{H}$ NMR $\left(500 \mathrm{MHz}, \mathrm{DMSO}-\mathrm{d}_{6}\right): \delta 3.72(3 \mathrm{H}, \mathrm{s}$, $\left.\mathrm{OCH}_{3}\right), 4.40(1 \mathrm{H}, \mathrm{s}, \mathrm{CH}), 6.87\left(2 \mathrm{H}, \mathrm{d}, J=8.1 \mathrm{~Hz}, \mathrm{H}_{\mathrm{Ar}}\right), 7.18$ $\left(2 \mathrm{H}, \mathrm{d}, J=8.1 \mathrm{~Hz}, \mathrm{H}_{\mathrm{Ar}}\right), 7.37\left(2 \mathrm{H}\right.$, br s, $\left.\mathrm{NH}_{2}\right), 7.45(1 \mathrm{H}$, $\left.\mathrm{d}, J=8.3 \mathrm{~Hz}, \mathrm{H}_{\mathrm{Ar}}\right), 7.49\left(1 \mathrm{H}, \mathrm{t}, J=7.8 \mathrm{~Hz}, \mathrm{H}_{\mathrm{Ar}}\right), 7.70(1 \mathrm{H}$, $\left.\mathrm{t}, J=7.7 \mathrm{~Hz}, \mathrm{H}_{\mathrm{Ar}}\right), 7.89\left(1 \mathrm{H}, \mathrm{d}, J=7.7 \mathrm{~Hz}, \mathrm{H}_{\mathrm{Ar}}\right) \mathrm{ppm} .{ }^{13} \mathrm{C}$ NMR $\left(125 \mathrm{MHz}, \mathrm{DMSO}_{-}\right.$): $\delta 55.90,59.10,105.13,113.84$, $114.71,117.37,120.18,123.29,125.47,129.64,133.66,136.26$, $152.94,153.94,158.79,159.20,160.38$ ppm. IR $\left(\mathrm{KBr}, \mathrm{cm}^{-1}\right)$ : $3378,3314,3190,2196,1709,1672,1608$. EI-MS: $\mathrm{m} / \mathrm{z}=$ 346.30.

2-Amino-4-(3,4-dimethoxyphenyl)-5-oxo-4,5dihydropyrano $[3,2-c]$ chromene-3-carbonitrile 4l. M.p. 226-228 $8^{\circ}$ [lit: 228-230 C] [21]. ${ }^{1} \mathrm{H}$ NMR $(500 \mathrm{MHz}$, DMSO-d 6 ): $\delta 3.70\left(6 \mathrm{H}, \mathrm{s}, 2 \mathrm{OCH}_{3}\right), 4.40(1 \mathrm{H}, \mathrm{s}, \mathrm{CH}), 6.75$ $\left(1 \mathrm{H}, \mathrm{d}, J=8.15 \mathrm{~Hz}, \mathrm{H}_{\mathrm{Ar}}\right), 6.86\left(2 \mathrm{H}, \mathrm{d}, J=8 \mathrm{~Hz}, \mathrm{H}_{\mathrm{Ar}}\right), 7.31$ $\left(2 \mathrm{H}\right.$, br s, $\left.\mathrm{NH}_{2}\right), 7.38\left(1 \mathrm{H}, \mathrm{d}, J=8.25 \mathrm{~Hz}, \mathrm{H}_{\mathrm{Ar}}\right), 7.43(1 \mathrm{H}$, $\left.\mathrm{t}, J=7.5 \mathrm{~Hz}, \mathrm{H}_{\mathrm{Ar}}\right), 7.64\left(1 \mathrm{H}, \mathrm{t}, J=7.65 \mathrm{~Hz}, \mathrm{H}, \mathrm{H}_{A r}\right), 7.88$ $\left(1 \mathrm{H}, \mathrm{d}, J=7.65 \mathrm{~Hz}, \mathrm{H}_{A r}\right)$ ppm. ${ }^{13} \mathrm{C}$ NMR (125 MHz, DMSO$\left.\mathrm{d}_{6}\right): \delta 36.51,55.50,55.56,58.27,104.08,111.78,111.96$, $112.97,116.40,119.22,119.67,122.38,124.47,132.68$, $135.82,148.02,148.58,152.05,153.11,157.93,159.49$ ppm. IR $\left(\mathrm{KBr}, \mathrm{cm}^{-1}\right): 3406,3326,2196,1710,1672,1609,1517$, 1378. EI-MS: $\mathrm{m} / \mathrm{z}=376.11$.

2-Amino-4-(2,5-dimethoxyphenyl)-5-oxo-4,5dihydropyrano[3,2-c]chromene-3-carbonitrile $4 \mathrm{~m}$. M.p. 246-248 C [lit: 247-248 $\mathrm{C}$ ] [11]. ${ }^{1} \mathrm{H}$ NMR (500 Hz,DMSO$\left.\mathrm{d}_{6}\right): \delta 3.63\left(3 \mathrm{H}, \mathrm{s}, \mathrm{OCH}_{3}\right), 3.64\left(3 \mathrm{H}, \mathrm{s}, \mathrm{OCH}_{3}\right), 4.64(1 \mathrm{H}$, $\mathrm{s}, \mathrm{CH}), 6.66\left(1 \mathrm{H}, \mathrm{d}, J=2.3 \mathrm{~Hz}, \mathrm{H}_{\mathrm{Ar}}\right), 6.75-6.79(1 \mathrm{H}, \mathrm{m}$, $\left.\mathrm{H}_{\mathrm{Ar}}\right), 6.90\left(1 \mathrm{H}, \mathrm{d}, J=8.8 \mathrm{~Hz}, \mathrm{H}_{\mathrm{Ar}}\right), 7.25\left(2 \mathrm{H}, \mathrm{s}, \mathrm{NH}_{2}\right)$, $7.41-7.49\left(2 \mathrm{H}, \mathrm{m}, \mathrm{H}_{\mathrm{Ar}}\right), 7.67\left(1 \mathrm{H}, \mathrm{t}, J=7.7 \mathrm{~Hz}, \mathrm{H}_{\mathrm{Ar}}\right), 7.89$ $\left(\mathrm{d}, J=7.7 \mathrm{~Hz}, 1 \mathrm{H}, \mathrm{H}_{\mathrm{Ar}}\right) \mathrm{ppm} .{ }^{13} \mathrm{C}$ NMR $(125 \mathrm{MHz}, \mathrm{DMSO}-$ $\left.\mathrm{d}_{6}\right): \delta 32.6,55.2,56.4,56.8,103.1,112.2,112.9,113.1,115.7$, $116.4,119.2,122.2,124.5,131.9,132.6,151.5,152.0,153.1$, $153.9,158.5,159.4 \mathrm{ppm}$. IR $\left(\mathrm{KBr}, \mathrm{cm}^{-1}\right): 3403,3322,3192$, $2195,1708,1672,1605,1501,1380$. EI-MS: $\mathrm{m} / \mathrm{z}=376.11$.

2-Amino-4-(3-nitrophenyl)-5-oxo-4,5-dihydropyrano[3,2-c] chromene-3-carbonitrile 4n. M.p. $258-260^{\circ} \mathrm{C}$ [lit: 262-264 ${ }^{\circ} \mathrm{C}$ ] [10]. ${ }^{1} \mathrm{H}$ NMR (500 MHz, DMSO-d 6 ): $\delta 4.64(1 \mathrm{H}, \mathrm{s}, \mathrm{CH})$, $7.38\left(1 \mathrm{H}, \mathrm{d}, J=6.7 \mathrm{~Hz}, \mathrm{H}_{\mathrm{Ar}}\right), 7.42\left(1 \mathrm{H}, \mathrm{t}, J=7.6 \mathrm{~Hz}, \mathrm{H}_{\mathrm{Ar}}\right)$, $7.68\left(2 \mathrm{H}\right.$, br s, $\left.\mathrm{NH}_{2}\right), 7.76\left(1 \mathrm{H}, \mathrm{t}, J=7.6 \mathrm{~Hz}, \mathrm{H}_{\mathrm{Ar}}\right), 7.45(1 \mathrm{H}$, $\left.\mathrm{dt}, J=7.5,1.3 \mathrm{~Hz}, \mathrm{H}_{\mathrm{Ar}}\right), 7.76\left(1 \mathrm{H}, \mathrm{d}, J=6.8 \mathrm{~Hz}, \mathrm{H}_{\mathrm{Ar}}\right), 7.82$ $\left(1 \mathrm{H}, \mathrm{dd}, J=6.8,1.2 \mathrm{~Hz}, \mathrm{H}_{\mathrm{Ar}}\right), 8.10(1 \mathrm{H}, \mathrm{dd}, J=8.4,1.4 \mathrm{~Hz}$, $\left.\mathrm{H}_{\mathrm{Ar}}\right), 8.16\left(1 \mathrm{H}, \mathrm{s}, \mathrm{H}_{\mathrm{Ar}}\right)$ ppm. ${ }^{13} \mathrm{C}$ NMR $(125 \mathrm{MHz}, \mathrm{DMSO}-$ $\left.\mathrm{d}_{6}\right): \delta 60.64,106.74,114.81,118.32,120.73,122.14,123.24$, $124.64,126.68,132.92,134.48,137.68,146.36,148.62,152.46$, $154.68,159.03,162.46 \mathrm{ppm}$. IR $\left(\mathrm{KBr}, \mathrm{cm}^{-1}\right): 3402,3328$, $3188,2196,1710,1676,1538,1346$. EI-MS: $\mathrm{m} / \mathrm{z}=361.07$.
2-Amino-4-(4-nitrophenyl)-5-oxo-4,5-dihydropyrano [3,2-c] chromene-3-carbonitrile 4o. M.p. 258-260 $\mathrm{C}$ [lit: 255-256 $\left.{ }^{\circ} \mathrm{C}\right]$ [10]. ${ }^{1} \mathrm{H}$ NMR $\left(500 \mathrm{MHz}, \mathrm{DMSO}-\mathrm{d}_{6}\right): \delta 4.66(1 \mathrm{H}, \mathrm{s}, \mathrm{CH})$, $7.45\left(1 \mathrm{H}, \mathrm{d}, J=8.3 \mathrm{~Hz}, \mathrm{H}_{\mathrm{Ar}}\right), 7.50\left(1 \mathrm{H}, \mathrm{t}, J=7.7 \mathrm{~Hz}, \mathrm{H}_{\mathrm{Ar}}\right)$, $7.56\left(2 \mathrm{H}\right.$, br s, $\left.\mathrm{NH}_{2}\right), 7.60\left(2 \mathrm{H}, \mathrm{d}, J=8.0 \mathrm{~Hz}, \mathrm{H}_{\mathrm{Ar}}\right), 7.76(1 \mathrm{H}$, $\left.\mathrm{t}, J=7.8 \mathrm{~Hz}, \mathrm{H}_{\mathrm{Ar}}\right), 7.92\left(1 \mathrm{H}, \mathrm{d}, J=7.8 \mathrm{~Hz}, \mathrm{H}_{\mathrm{Ar}}\right), 8.16(2 \mathrm{H}$, $\left.\mathrm{d}, J=8.3 \mathrm{~Hz}, \mathrm{H}_{\mathrm{Ar}}\right)$ ppm. ${ }^{13} \mathrm{C}$ NMR $\left(125 \mathrm{MHz}, \mathrm{DMSO}-\mathrm{d}_{6}\right): \delta$ 59.65, 104.64, 114.74, 117.68, 119.78, 123.43, 124.57, 126.56, $131.04,133.99,148.46,152.61,153.13,154.81,158.93$, $161.42 \mathrm{ppm}$. IR $\left(\mathrm{KBr}, \mathrm{cm}^{-1}\right): 3482,3432,3371,3340,2193$, $1715,1673,1606,1508,1372$, 1304. EI-MS: $\mathrm{m} / \mathrm{z}=361.07$.

2-Amino-4-(4-hydroxyphenyl)-5-oxo-4,5-dihydropyrano[3,2c]chromene-3-carbonitrile 4p. M.p. 259-260 C [lit: 261$262^{\circ} \mathrm{C}$ ] [24]. ${ }^{1} \mathrm{H}$ NMR $\left(500 \mathrm{MHz}, \mathrm{DMSO}-\mathrm{d}_{6}\right): \delta 9.53(1 \mathrm{H}, \mathrm{s}$, $\mathrm{OH}), 4.46(1 \mathrm{H}, \mathrm{s}, \mathrm{CH}), 6.87\left(2 \mathrm{H}, \mathrm{d}, J=8.1 \mathrm{~Hz}, \mathrm{H}_{\mathrm{Ar}}\right), 7.18$ $\left(2 \mathrm{H}, \mathrm{d}, J=8.1 \mathrm{~Hz}, \mathrm{H}_{\mathrm{Ar}}\right), 7.46\left(2 \mathrm{H}\right.$, br s, $\left.\mathrm{NH}_{2}\right), 7.45(1 \mathrm{H}$, $\left.\mathrm{d}, J=8.3 \mathrm{~Hz}, \mathrm{H}_{\mathrm{Ar}}\right), 7.49\left(1 \mathrm{H}, \mathrm{t}, J=7.8 \mathrm{~Hz}, \mathrm{H}_{\mathrm{Ar}}\right), 7.70(1 \mathrm{H}$, $\left.\mathrm{t}, J=7.7 \mathrm{~Hz}, \mathrm{H}_{\mathrm{Ar}}\right), 7.89\left(1 \mathrm{H}, \mathrm{d}, J=7.7 \mathrm{~Hz}, \mathrm{H}_{\mathrm{Ar}}\right) \mathrm{ppm} .{ }^{13} \mathrm{C}$ NMR (125 MHz, DMSO-d ${ }_{6}$ ): $\delta 55.90,59.10,105.13,113.84$, $114.71,117.37,120.18,123.29,125.47,129.64,133.66,136.26$, $152.94,153.94,158.79,159.20,160.38 \mathrm{ppm}$. IR $\left(\mathrm{KBr}, \mathrm{cm}^{-1}\right)$ : $3378,3314,3190,2196,1710,1672,1608$. EI-MS: $\mathrm{m} / \mathrm{z}=$ 332.03 .

\section{Acknowledgments}

The authors thank the Managing Trustee and the Director of Sankar Foundation for their financial support and encouragement.

\section{References}

[1] G. R. Geen, J. M. Evans, and A. K. Vong, "5.09—pyrans and their benzo derivatives: applications," Comprehensive Heterocyclic Chemistry II, vol. 5, pp. 469-500, 1996.

[2] J.-C. Jung and O.-S. Park, "Synthetic approaches and biological activities of 4-hydroxycoumarin derivatives," Molecules, vol. 14, no. 11, pp. 4790-4803, 2009.

[3] A. M. M. El-Saghier, M. B. Naili, B. K. Rammash, N. A. Saleh, and K. M. Kreddan, "Synthesis and antibacterial activity of some new fused chromenes," ARKIVOC, vol. 2007, no. 16, pp. 83-91, 2007.

[4] L. Kováčiková, R. Gašparová, A. Boháč, M. Ďurana, and M. Lácová, "Synthesis of 3-phenyl-2H,5H-pyrano[3,2-c]chromen2-one derivatives and their antineoplastic activity," ARKIVOC, vol. 2010, no. 11, pp. 188-203, 2010.

[5] C. B. Sangani, D. C. Mungra, M. P. Patel, and R. G. Patel, "Synthesis and antimicrobial screening of pyrano[3,2-c]chromene derivatives of $1 H$-pyrazoles ," Central European Journal of Chemistry, vol. 9, no. 4, pp. 635-647, 2011.

[6] A. Tanabe, H. Nakashima, O. Yoshida, N. Yamamoto, O. Tenmyo, and T. Oki, "Inhibitory effect of new antibiotic, pradimicin A on infectivity, cytopathic effect and replication of human immunodeficiency virus in vitro," The Journal of Antibiotics, vol. 41, no. 11, pp. 1708-1710, 1988.

[7] T. A. Bayer, S. Schäfer, H. Breyhan, O. Wirths, C. Treiber, and G. Multhaup, "A vicious circle: role of oxidative stress, intraneuronal Abeta and $\mathrm{Cu}$ in Alzheimer's disease," Clinical Neuropathology, vol. 25, no. 4, pp. 163-171, 2006. 
[8] R. M. Shaker, "Synthesis and reactions of some new $4 H$ pyrano[3,2-c] benzopyran-5-one derivatives and their potential biological activities," Pharmazie, vol. 51, no. 3, pp. 148-151, 1996.

[9] M. Kidwai and S. Saxena, "Convenient preparation of pyrano benzopyranes in aqueous media," Synthetic Communications, vol. 36, no. 18, pp. 2737-2742, 2006.

[10] S. Abdolmohammadi and S. Balalaie, "Novel and efficient catalysts for the one-pot synthesis of 3,4-dihydro-pyrano $[c]$ chromene derivatives in aqueous media," Tetrahedron Letters, vol. 48, no. 18, pp. 3299-3303, 2007.

[11] A. Shaabani, S. Samadi, Z. Badri, and A. Rahmati, "Ionic liquid promoted efficient and rapid one-pot synthesis of pyran annulated heterocyclic systems," Catalysis Letters, vol. 104, no. 1-2, pp. 39-43, 2005.

[12] H. R. Shaterian and A. R. Oveisi, "A simple green approach to the synthesis of 2-amino-5-oxo-4,5-dihydropyrano[3,2c]chromene-3-carbonitrile derivatives catalyzed by 3hydroxypropanaminium acetate (HPAA) as a new ionic liquid," Journal of the Iranian Chemical Society, vol. 8, no. 2, pp. 545-552, 2011.

[13] A. K. Bose, S. Pednekar, S. N. Ganguly, G. Chakraborty, and M. S. Manhas, "A simplified green chemistry approach to the Biginelli reaction using 'Grindstone Chemistry," Tetrahedron Letters, vol. 45, no. 45, pp. 8351-8353, 2004.

[14] W. T. Richards and A. L. Loomis, "The chemical effects of high frequency sound waves I. A preliminary survey," Journal of the American Chemical Society, vol. 49, no. 12, pp. 3086-3100, 1927.

[15] M. Blaskovicova, A. Gaplovsky, and J. Blasko, "Synthesis and photochemistry of 1-iodocyclohexene: influence of ultrasound on ionic vs. radical behaviour," Molecules, vol. 12, no. 2, pp. 188-193, 2007.

[16] X. K. Wang, G. H. Chen, and W. L. Guo, "Sonochemical degradation kinetics of methyl violet in aqueous solutions," Molecules, vol. 8, no. 1, pp. 40-44, 2003.

[17] V. Singh, K. P. Kaur, A. Khurana, and G. L. Kad, "Ultrasound: a boon in the synthesis of organic compounds," Resonance, vol. 3 , no. 9, pp. 56-60, 1998.

[18] J. V. Prasad, J. S. Reddy, N. Ravikumar, K. A. Solomon, and G. Gopikrishna, "An efficient ultrasound promoted catalyst-free protocol for the synthesis of chromeno[4,3-b]quinolin-6-ones," Journal of Chemical Sciences, vol. 123, no. 5, pp. 673-679, 2011.

[19] M. L. Kantam, C. V. Rajasekhar, G. Gopikrishna, K. R. Reddy, and B. M. Choudary, "Proline catalyzed two-component, threecomponent and self-asymmetric Mannich reactions promoted by ultrasonic conditions," Tetrahedron Letters, vol. 47, no. 33, pp. 5965-5967, 2006.

[20] D.-Q. Shi, J. Wang, Q.-Y. Zhuang, and X.-S. Wang, "Threecomponent one-pot synthesis of 1,6-dioxa-5-oxo-1,4,5,6tetrahydrophenanthrene derivatives in aqueous media," Chinese Journal of Organic Chemistry, vol. 26, no. 5, pp. 643-647, 2006.

[21] J. M. Khurana and S. Kumar, "Tetrabutylammonium bromide (TBAB): a neutral and efficient catalyst for the synthesis of biscoumarin and 3,4-dihydropyrano $[c]$ chromene derivatives in water and solvent-free conditions," Tetrahedron Letters, vol. 50, no. 28, pp. 4125-4127, 2009.

[22] M. M. Heravi, B. A. Jani, F. Derikvand, F. F. Bamoharram, and H. A. Oskooie, "Three component, one-pot synthesis of dihydropyrano $[3,2-c]$ chromene derivatives in the presence of $\mathrm{H}_{6} \mathrm{P}_{2} \mathrm{~W}_{18} \mathrm{O}_{62} \cdot 18 \mathrm{H}_{2} \mathrm{O}$ as a green and recyclable catalyst," Catalysis Communications, vol. 10, no. 3, pp. 272-275, 2008.
[23] H. Mehrabi and H. Abusaidi, "Synthesis of biscoumarin and 3,4-dihydropyrano[c]chromene derivatives catalysed by sodium dodecyl sulfate (SDS) in neat water," Journal of the Iranian Chemical Society, vol. 7, no. 4, pp. 890-894, 2010.

[24] X.-S. Wang, Z.-S. Zeng, D.-Q. Shi, X.-Y. Wei, Z.-M. Zong, “Onepot synthesis of 2-amino-4-aryl-4H-pyrano[3,2-c]coumarin Derivatives using $\mathrm{KF} / \mathrm{Al}_{2} \mathrm{O}_{3}$ as catalyst," Chinese Journal of Organic Chemistry, vol. 25, no. 9, pp. 1138-1141, 2005. 

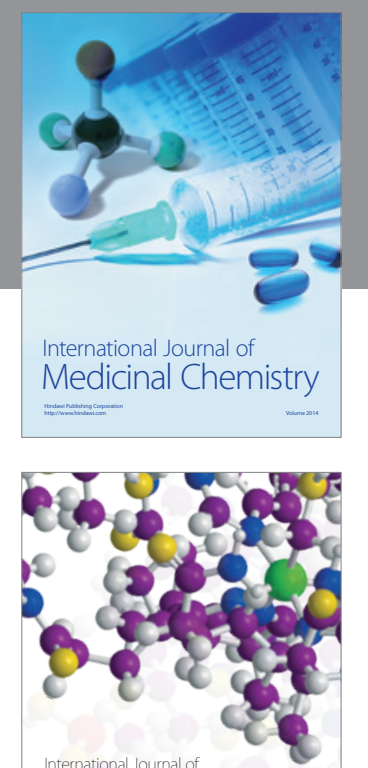

\section{Carbohydrate} Chemistry

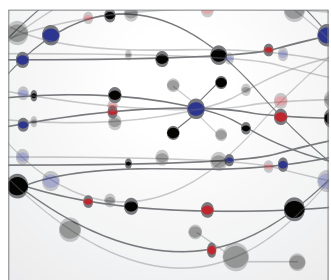

The Scientific World Journal
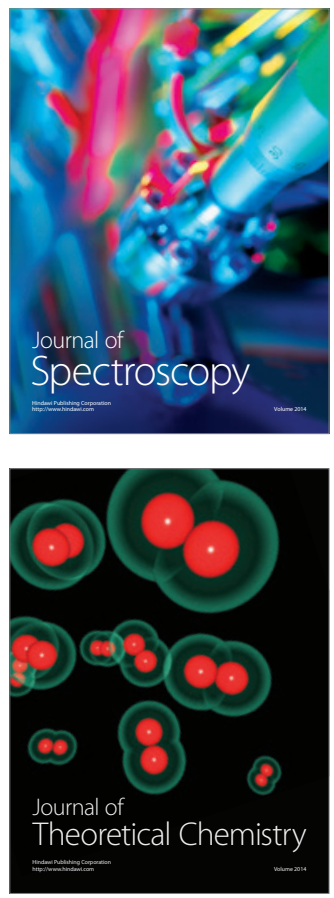
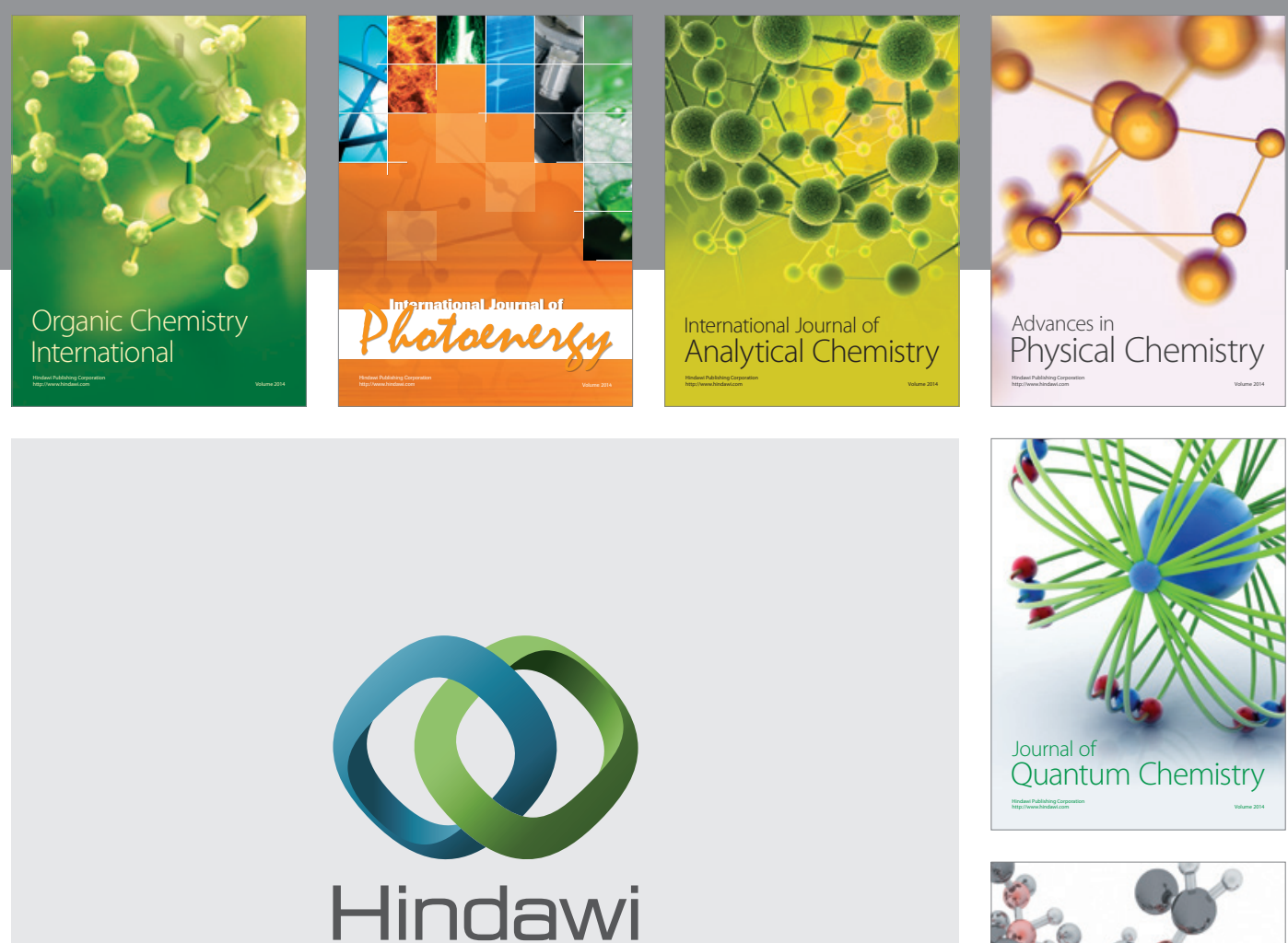

Submit your manuscripts at

http://www.hindawi.com

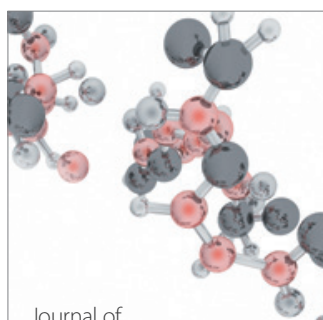

Analytical Methods

in Chemistry

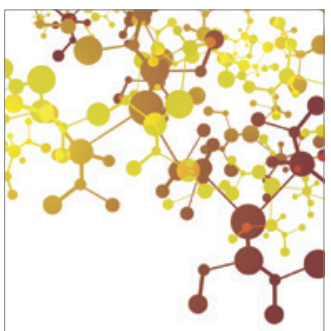

Journal of

Applied Chemistry

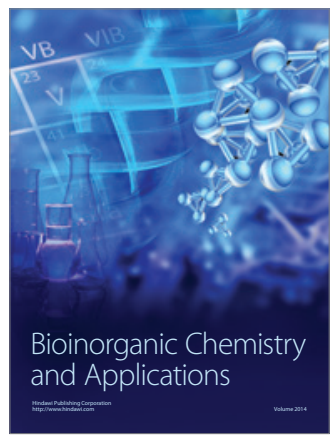

Inorganic Chemistry
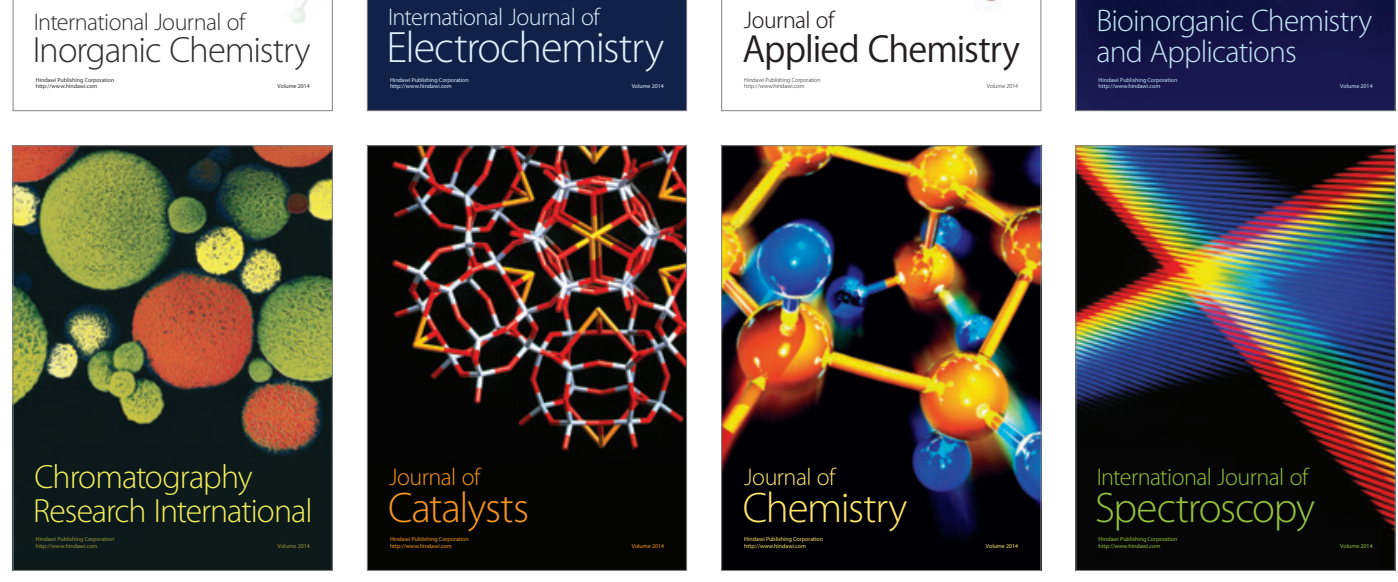